\title{
Monte Carlo Analysis of the Influence of Different Packaging on MOSFET Energy Response to X-rays and Gamma Radiation
}

\author{
S.J. StankoviĆ ${ }^{a, *}$, R.D. IliĆ ${ }^{a}$, M. ŽivanoviĆ ${ }^{a}$, K.S. JankOViĆ ${ }^{b}$ AND B. LonČAR ${ }^{c}$ \\ ${ }^{a}$ Vinča Institute of Nuclear Sciences, Belgrade, Serbia \\ ${ }^{b}$ Institute for Testing Materials-IMS, Belgrade, Serbia \\ ${ }^{c}$ Faculty of Technology and Metallurgy, University of Belgrade, Belgrade, Serbia
}

(Received September 5, 2011; in final form July 3, 2012)

\begin{abstract}
Radiation sensing MOSFETs have found numerous applications as detectors or device components in radiation fields used in nuclear industry, medical applications and space research. Monte Carlo simulations of MOSFET energy response to X-ray and gamma radiation for different packaging were performed. The photon transport Monte Carlo software FOTELP-2K10 has been adapted to obtain the energy deposited in MOSFET structure with microscopic dimensions. In this work the ratio between values of total energy deposited in the sensitive volume (thick $\mathrm{SiO}_{2}$ layer) for cases of MOSFET structure with and without package lid is presented. For this purpose is defined the shielding energy dependence factor (SDEF), and gave its value for kovar and Ti-24Al-11Nb and Ti-13Nb-13Zr alloys as lid materials.
\end{abstract}

PACS: $07.85 . \mathrm{Fv}, 85.30 . \mathrm{Tv}, 87.55 . \mathrm{kh}$

\section{Introduction}

In recent years an increasing number of research works have been addressed to the study of the characteristics and applications of MOSFET detectors in the field of nuclear industry, medical applications and space research. A MOSFET first used as a radiation dosimeter was demonstrated by Holmes-Siedle in 1974 [1]. For ionizing dose monitoring in an unknown radiation field the response of the sensor should be based on the effect of degradation caused by ionizing processes in the gate oxide [2]. The main characteristics of these detectors are the small size and on-line readout possibility. However, MOSFETs have been limited in dependence of total absorbed dose on incident photon energy. In recently published paper, it has been experimentally studied energy dependence of MOSFET dosimeters over the photon energy range from $14 \mathrm{keV}$ to $1250 \mathrm{keV} \mathrm{[3].} \mathrm{An-}$ other group of researchers carried out the experiments in field of a dosimetric X-rays unit, and a radiotherapeutic linear accelerator up to $8 \mathrm{MeV}$ [4]. The first tests with synchrotron radiation were done by Kron et al. [5] who studied the energy dependence of MOSFETs in free-air geometry by using a monoenergetic, large field size X-ray beam. Monte Carlo simulations of the energy response

\footnotetext{
* corresponding author; e-mail: srbas@vinca.rs
}

of a conventionally packed single MOSFET detector were performed with the goal of improving MOSFET energy dependence for personal accident and military dosimetry [6]. One of a major goal of our work was the presentation of the shielding energy dependence factor (SDEF) which assesses the impact of lid with different shielding materials (kovar and titanium alloys) to MOSFET response for different photon energy. In doing so, the established numerical method for analysis influence of the packaging to energy dependence, was carried out prior to any experimental testing.

\section{Theory}

The sensitive element of the MOSFET detector is the gate oxide, which is defined by a thin silicon oxide layer positioned on top of a thick silicon substrate (see Fig. 1). Radiation creates charge within the thin silicon dioxide layer and this charge and its long term trapping leads to a change of threshold voltage of the MOSFET transistor. In the case of MOSFET, the threshold voltage shift produced by the photon irradiation can be expressed by the following simple model [4]:

$$
\Delta V=-\frac{q}{\varepsilon_{\mathrm{ox}}} g f_{\mathrm{R}} f_{\mathrm{T}}\left(d_{\mathrm{ox}}-x_{\mathrm{C}}\right)^{2} D\left(\mathrm{SiO}_{2}, E_{\mathrm{p}}\right),
$$

where $q$ is electron charge, $\varepsilon_{\mathrm{ox}}$ the dielectric constant for the silica, $g$ the electron-hole pair generation, $f_{\mathrm{R}}$ the 
probability of a hole to escape recombination, $f_{\mathrm{T}}$ the probability of a hole to be trapped in a tin sheet at the distance $x_{\mathrm{C}}$ from the $\mathrm{Si} / \mathrm{SiO}_{2}$ interface, $d_{\mathrm{ox}}$ the thickness of the oxide layer and $D\left(\mathrm{SiO}_{2}, E_{\mathrm{p}}\right)$ the absorbed dose in the gate oxide $\mathrm{SiO}_{2}$ and $E_{\mathrm{p}}$ is photon energy. Absorbed dose is in relation to deposited energy in zone $\mathrm{SiO}_{2}$ as

$$
D\left(\mathrm{SiO}_{2}, E_{\mathrm{p}}\right)=\frac{E_{\mathrm{D}}\left(\mathrm{SiO}_{2}, E_{\mathrm{p}}\right)}{\rho_{\mathrm{SiO} 2} K_{\mathrm{SiO} 2}},
$$

where $\rho_{\mathrm{SiO} 2}$ and $K_{\mathrm{SiO} 2}$ are density and volume of zone $\mathrm{SiO}_{2}$.

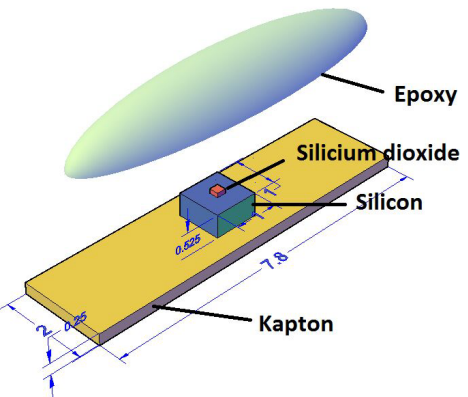

Fig. 1. Schematic diagram of the MOSFET dosimeter without package lid. Sensitive volume $\left(\mathrm{SiO}_{2}\right)$ is located on top of the silicon substrate and under the epoxy cover.

The bare MOSFET is encapsulated in epoxy glue which mechanically protects the chip. To trace the influence of lid is the one intended by the physical protection, on the other hand it can also be used for protection against radiation, is introduced that defines the shielding energy dependence factor (SDEF) for different photon energies and for different constructional materials of lid

$$
\operatorname{SDEF}\left(E_{\mathrm{p}}\right)=\frac{D\left(\mathrm{SiO}_{2}, E_{\mathrm{p}}\right)_{\text {shield }}}{D\left(\mathrm{SiO}_{2}, E_{\mathrm{p}}\right)_{\text {bare }}} .
$$

Factor SDEF is defined as the ratio of value absorbed dose, which in fact means that it is equal to the energy deposited when the MOSFET is shielded with protection, and the MOSFET without lid.

When we know the energy dependence factor for SDEF MOSFET with and without armor, there can be carried out the analysis of whether and to what extent the energy required compensates the electronic components.

\section{Geometry and materials}

In order to use numerical methods in this paper, the appropriate geometry form of the MOSFET dosimeter was defined using adequate software. FOTELP-2K10 code $[7,8]$ uses RFG and PENGEOM6 software modules for dosimeter geometry description. According to the available data for a very sensitive MOSFET $[9,10]$, a silicon substrate $\left(1 \mathrm{~mm}^{2}\right.$ in area, $0.525 \mathrm{~mm}$ thick $)$ is contained within a $1 \mathrm{~mm}$ thick epoxy bulb (Fig. 1). The silicon substrate and the epoxy bulb are attached to the end of a flexible kapton cable $(0.25 \mathrm{~mm}$ thick, $2 \mathrm{~mm}$ wide) encapsulating two gold wires. The sensitive volume $\left(0.2 \mathrm{~mm} \times 0.2 \mathrm{~mm}\right.$ in area) is a $1 \mu \mathrm{m}$ thick $\mathrm{SiO}_{2}$ layer. It is sandwiched between the epoxy bulb and the silicon substrate. It is noted that the dimensions of the Monte Carlo dosimeter model are as accurate as the information provided by the manufacturer. The only uncertainty in the geometry is the shape of the epoxy. Although the actual shape may differ from the semi-ellipsoid, as simulated in the Monte Carlo model, its impact on the dose is very small, due to the character of the our numerical experiment based on photon transport. Package lid is a $250 \mu \mathrm{m}$ thick protection shell over $250 \mu \mathrm{m}$ vacuum layer, which is up epoxy bulb. Three different materials for protection shell are: 1. kovar (Ni, Co, Fe), 2. Ti-24Al-11Nb, 3. Ti-13Nb-13Zr.

\section{Numerical method}

Characterization of radiation sensing MOSFET by Monte Carlo method was made with FOTELP-2K10 code for incident photons with different energies. FOTELP$-2 \mathrm{~K} 10$ code has great competency in successful resolving of radiation transport problems which review interactions of gamma and X-rays with electronic components and devices, such as MOSFET dosimeters [9, 11], CdZnTe detector [12], diamond detector [13] and silicon carbide detector [14]. Physical rigor is maximized by employing the best available cross-sections and high speed routines for random values sampling from their distributions, and the most complete physical model for describing the transport and production of the photon/electron/positron cascade from $100.0 \mathrm{MeV}$ down to $1.0 \mathrm{keV}$. FOTELP-2K10 is developed for numerical experiments by Monte Carlo techniques for dosimetry, radiation damage, radiation therapy and other actual applications of these particles. For the photon history, the trajectory is generated by following it from scattering to scattering using corresponding inverse distribution between collisions, types of target, types of collisions, types of secondaries, their energy and scattering angles. Photon interactions are coherent scattering, incoherent scattering, photoelectric absorption and pair production. The Doppler broadening in the Compton scattering is taken. The histories of secondary photons include bremsstrahlung and positronelectron annihilation radiation. The condensed history Monte Carlo method is used for the electron and positron transport simulation.

During a history the particles lose energy in collisions, and the secondary particles are generated on the step according to the probabilities for their occurrence. Electron (positron) energy loss is through inelastic electronelectron $\left(\mathrm{e}^{-}, \mathrm{e}^{-}\right)$and positron-electron $\left(\mathrm{e}^{+}, \mathrm{e}^{-}\right)$collisions and bremsstrahlung generation. The fluctuation of energy loss (straggling) is included according to the Landau or Blunk-Westphal distributions with 9 Gaussians. The secondary electrons, which follow history of particles, include knock-on, pair production, Compton and photoelectric electrons. The secondary positrons, which 
follow pair production, are included, too. With atomic data, the electron and positron Monte Carlo simulation is broadened to treat atomic ion relaxation after photoeffect and impact ionization. Flexibility of the codes permits them to be tailored to specific applications and allows the capabilities of the codes to be extended to more complex applications, especially in radiotherapy in voxelized geometry using CT data. The actual version of FOTELP-2K10 program was expanded by adding new routines for accepting from XCOM [15] data for photon interaction, adapted routines for coherent and incoherent scattering and annihilation, and PENGEOM6 package for geometry modeling. In this way, Monte Carlo simulations were being improved, particularly at low energies that are typical for simulations in radiation and nuclear physics and technology.

\section{Results and discussion}

Although the energy response of MOS devices and their packaging to $\mathrm{X}$ and gamma radiation has previously been studied experimentally and analytically, until recently there have been no investigations of energy response in a way characteristic and necessary for optimization methodology in semiconductor components design. Results of calculations for deposited energy with Monte Carlo simulations for $10^{7}$ photons per one simulation, presented in Figs. 2 to 5 . In each simulation, the primary photon beam and the pencil beam has a direction such that the beam axis is perpendicular to the upper horizontal surface of $\mathrm{SiO}_{2}$ zone. In Table we presented data for shielding energy dependence factor for three materials: kovar, titanium alloy with aluminum and niobium, and for titanium alloy with zirconium and niobium.

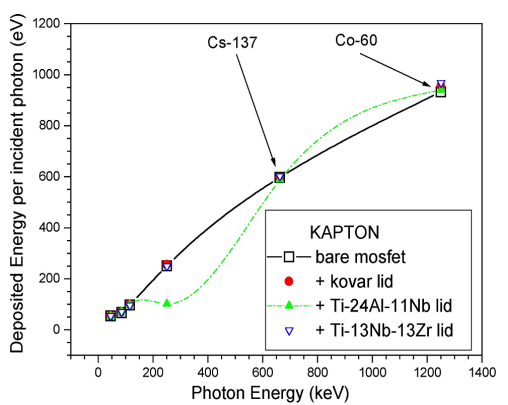

Fig. 2. Energy dependence for material zone kapton.

Significant differences between the average atomic numbers of the materials of a MOSFET transistor chip and its package of kovar or titanium alloys, should produce a different energy response. Materials as kapton (Fig. 2) and epoxy (Fig. 5) have a similar trend in the energy dependence of radiation because of similar characteristics, such as for example the effective cross-section for interacting these materials with photons. In the case for silicon (Fig. 3) and gate oxide $\left(\mathrm{SiO}_{2}\right)$ (Fig. 4), at lower photon energies of $150 \mathrm{keV}$ there is a sudden increase in

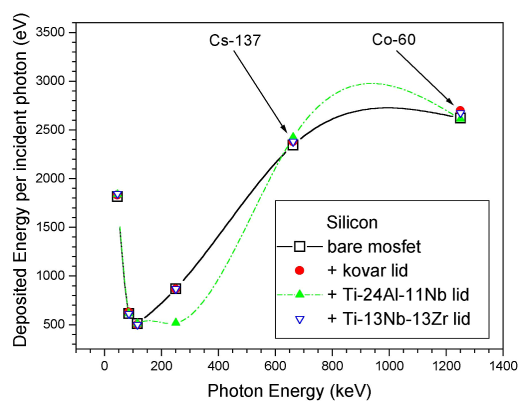

Fig. 3. Energy dependence for material zone silicon.

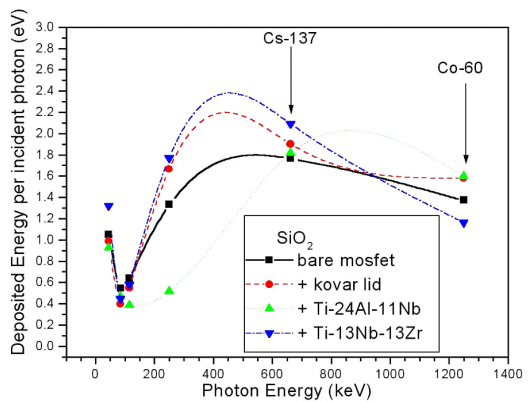

Fig. 4. Energy dependence for gate oxide $\left(\mathrm{SiO}_{2}\right)$.

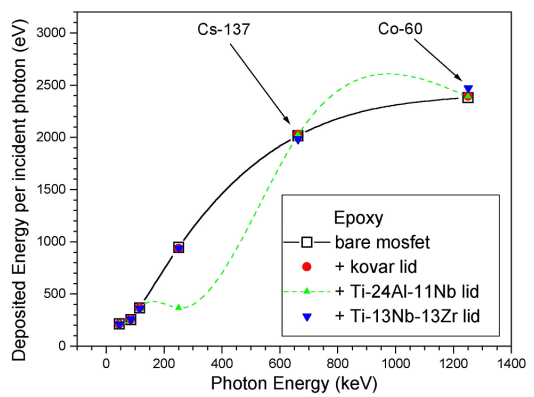

Fig. 5. Energy dependence for material zone epoxy.

TABLE

Shielding energy dependence factor SDEF for kovar and titanium alloys.

\begin{tabular}{c|c|c|c}
\hline \hline $\begin{array}{c}\text { Photon } \\
\text { energy } \\
{[\mathrm{keV}]}\end{array}$ & SDEF $_{\text {kovar }}$ & SDEF $_{\text {TiAlNb }}$ & SDEF $_{\text {TiNbZr }}$ \\
\hline 45 & 0.939 & 0.880 & 1.257 \\
85 & 0.735 & 0.856 & 0.821 \\
115 & 0.860 & 0.605 & 0.909 \\
250 & 1.251 & 0.387 & 1.330 \\
662 & 1.077 & 1.029 & 1.185 \\
1250 & 1.152 & 1.163 & 0.847
\end{tabular}


the effect of deposited energy in the material of titanium alloys and kovar, which requires construction solutions which perform the energy compensation. As for the energy dependence of photon energy greater than $150 \mathrm{keV}$ titanium alloy with zirconium is an acceptable absorption characteristics similar to the kovar. Titanium alloy with aluminum is considerably less suitable for shielding function. Although all three materials for lid have similar behavior of separate functions for the energy dependence, there are very significant differences in their values SDEF (see Table.).

In the case of kovar for the energy range from $45 \mathrm{keV}$ to Co-60 energies (two lines, $1.17 \mathrm{MeV}$ and $1.33 \mathrm{MeV}$ ) the energy compensation is necessary for less than $30 \%$. The materials of two titanium alloys have a deviation up to $30 \%$ in values SDEF for most energies. However it is worth to note that major problems arise with the alloy titanium aluminum alloy on energies $115 \mathrm{keV}$ and $250 \mathrm{keV}$.

\section{Conclusion}

Observations indicate that the MOSFET response is dependent on photon energy as well as varying with the package configuration. The photon transport Monte Carlo code FOTELP-2K10 has been adopted to analyze the influence of the MOSFET package on its energy response (see results presented in Figs. 2 to 6 ). The packaging effect was investigated by comparing the total energy deposited in zones of MOSFET structure. In this work we presented the ratio between values of total energy deposited in the sensitive volume (thick $\mathrm{SiO}_{2}$ layer) for cases of MOSFET structure with and without package lid. For this purpose we define the SDEF, and give its value for kovar and Ti-24Al-11Nb and Ti-13Nb-13Zr alloys as lid materials. Further, we perform identification of dependence of total energy deposited with varying of photon energy, for all zones of interest with specially selected type of MOSFET.

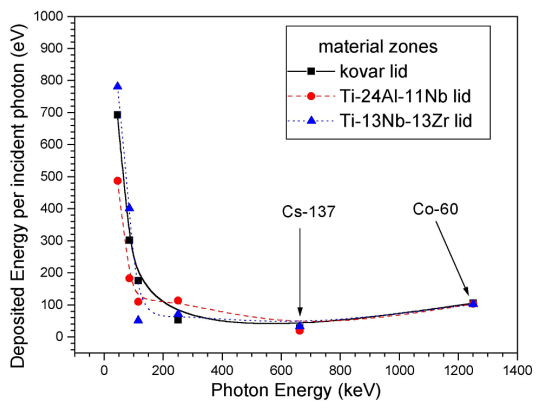

Fig. 6. Energy dependence for kovar and titanium alloys.

Our intention in this paper was, among other things, to initiate ideas on how to set up appropriate experiments, which would follow conditions imposed on numerical simulations. However, the problem of electrical measurements of properties in medium that has microscopic (or even less) dimensions is well known. Thus, the purpose of this paper is to present the possibilities of the numerical simulations for the deposited energy distribution on microscopic or submicroscopic levels, primarily in qualitative sense. Further investigations in other areas of interest, related to the study of electrical and technological characteristics of components necessary in the modern semiconductor technique and material physics, are planned.

\section{Acknowledgments}

This work was provided under financial support by the Ministry of Education and Science, Republic of Serbia, under projects 171007,36017 , and 43009 .

\section{References}

[1] A. Holmes-Siedle, Nucl. Instrum. Methods 121, 169 (1974).

[2] A.B. Rosenfeld, M.I. Reinhard, D. Marinaro, P. Ihnat, G. Taylor, L. Peak, N. Freeman, D. Alexiev, M. Lerch, IEEE Trans. Nuclear Sci. 46, 1766 (1999).

[3] G.J. Brucker, S. Kronenberg, F. Gentner, IEEE Trans. Nucl. Sci. 42, 33 (1995).

[4] Z. Savic, M. Kovačević, M. Petrović, Radiat. Protect. Dosim. 64, 205 (1996).

[5] T. Kron, L. Duggan, A. Rosenfeld, T. Smith, M. Butson, G. Kaplan, S. Howlet, K. Hyodo, Phys. Med. Biol. 43, 3235 (1998).

[6] M.A.R. Othman, D.L. Cutajar, N. Hardcastle, S. Guatelli, A.B. Rosenfeld, Radiat. Prot. Dosim. 141, 10 (2010).

[7] FOTELP-2K6, Photons, Electrons and Positrons Transport in $3 D$ by Monte Carlo Techniques, IAEA-1388, http://www.nea.fr/tools/abstract/ detail/iaea1388.

[8] FOTELP-2K10, http://www.vinca.rs/\%7Erasa/ doc/FOTPEN10.PDF

[9] S.J. Stanković, R.D. Ilić, P. Osmokrović, B. Lončar, A. Vasić, IEEE Trans. Plasma Sci. 34, 1715 (2006).

[10] E.A. Siegbahn, E. Bräuer-Krisch, A. Bravin, H. Nettelbeck, M.L.F. Lerch, A.B. Rosenfeld, Med. Phys. 36, 1128 (2009).

[11] S.J. Stanković, R.D. Ilić, M. Petrović, B. Lončar, A. Vasić, Mater. Sci. Forum 518, 361 (2006).

[12] S.J. Stanković, M. Petrović, M. Kovačević, A. Vasić, P. Osmokrović, B. Lončar, Mater. Sci. Forum $\mathbf{5 5 5}$ 141 (2007).

[13] S.J. Stanković, R.D. Ilic, D.M. Davidovic, M. Petrovic, Acta Phys. Pol. A 115, 816 (2009).

[14] S.J. Stanković, R.D. Ilic, K.S. Jankovic, A Vasic-Milovanovic, B. Loncar, Acta Phys. Pol. A 120, 252 (2011).

[15] XCOM: Photon Cross Sections Database, 2010, http://physics.nist.gov/PhysRefData/Xcom/ $\mathrm{html} / \mathrm{x} \operatorname{com} 1 . \mathrm{html}$. 